\title{
Modification of Carbohydrate Content in Developing Tomato Fruit
}

\author{
Arthur A. Schaffer ${ }^{1}$, Marina Petreikov, Daphne Miron², Miriam Fogelman, Moshe Spiegelman, \\ Zecharia Bnei-Moshe, and Shmuel Shen \\ Department of Vegetable Crops, ARO-Volcani Center, Bet Dagan, Israel \\ David Granot, Rivka Hadas, and Nir Dai \\ Department of Field Crops, ARO-Volcani Center, Bet Dagan, Israel
Ilan Levin, Moshe Bar ${ }^{3}$, Michael Friedman, Meir Pilowsky, Nehama Gilboa, and Leah Chen Department of Plant Genetics, ARO-Volcani Center, Bet Dagan, Israel

\begin{abstract}
The carbohydrate content of the tomato fruit is a major determinant of the quality and value of the crop, whether it be for the fresh produce market or for processing. Soluble sugar levels contribute strongly to the soluble solids content and to tomato flavor (Stevens et al., 1977a, 1977b) and, therefore, increasing these levels has been the goal of many research efforts.

The carbohydrate economy of developing tomato fruit is determined by the whole gamut of plant source-sink relationships. These include photoassimilate production at the source, its partitioning within the leaf, transport and export to alternative sinks and, finally, import into and metabolism within the fruit sink. Although the carbohydrate status of the fruit is a product of the interactions among all these processes, the fate of imported photoassimilate partitioned to the fruit is controlled only by the carbohydrate metabolism in the fruit itself.
\end{abstract}

Clearly, fruit carbohydrate content can be increased by improving the source contribution to the carbohydrate economy. This can be seen, for example, in the effects on fruit total soluble solids (TSS) content of: light intensity; plant architecture modifications, such as by pruning; or genetic control of growth habit ( $s p$ gene) (see Davies and Hobson, 1981). The same is likely to apply to the effects of the physiological components of loading, transport, and unloading processes on fruit sugar content. Recent studies of these processes in tomatoes (Ruan and Patrick, 1995; Ruan et al., 1997) should help to set the stage for the

Received for publication 15 Oct. 1998. Accepted for publication 17 Nov. 1998. This paper is dedicated to the memory of Moshe Spiegelman. The research was partially supported by grant no. 2451-94 from the United States-Israel Binational Agricultural Research and Development (BARD) Fund. This is contribution no. 2295-E, 1997 series, from the Agricultural Research Organization, The Volcani Center, Bet Dagan, Israel. The cost of publishing this paper was defrayed in part by the payment of page charges. Under postal regulations, this paper therefore must be hereby marked advertisement solely to indicate this fact. ${ }^{1}$ To whom reprint requests should be sent; e-mail: vcaris@ volcani.agri.gov.il ${ }^{2}$ Present address: Dept. of Plant Genetics, Weizmann Institute of Science, Rehovot, Israel.

${ }^{3}$ Present address: Zeraim Seed Co., Gedera, Israel. modulation of these steps in order to impact on fruit carbohydrate levels.

The goal of this paper is to address the role of tomato fruit metabolism in determining the carbohydrate content of the fruit. Since the fate of a translocated sucrose molecule after it passes the fruit pedicel will be determined by sink metabolism, modifying fruit carbohydrate metabolism should affect fruit carbohydrate composition. More questionable is the hypothesis that such modifications could also increase the total carbohydrate import into, and the subsequent content of, the fruit.

Within Lycopersicon sp. there is a broad range of genetic variability for fruit carbohydrate metabolism. This includes sucrose accumulation and modified ratios of fructose to glucose in the mature fruit, as well as increased starch synthesis in the immature fruit. This genetic variability is being utilized to develop tomato genotypes with modified carbohydrate metabolism and accumulation patterns; this allows us to begin to address the relationship between modified fruit carbohydrate metabolism and the carbon economy of the fruit.

\section{CONTRIBUTION OF SUGARS TO CARBON BUDGET OF TOMATO FRUIT}

The subject of tomato fruit development, especially as it relates to fruit carbon economy, has recently been reviewed by Ho (1996a, 1996b). In this paper, only a brief review of some key points relating to our research on the subject will be presented.

The hexose sugars, glucose and fructose, in an approximately equimolar ratio, make up $\approx 50 \%$ of the dry matter of the mature fruit (Davies and Hobson, 1981). A relatively small portion of the soluble sugars, generally $<5 \%$, consists of sucrose, in spite of the fact that sucrose is the sugar translocated into the fruit (Walker and Ho, 1977).

The tomato fruit is at times described as undergoing a phase of rapid hexose accumulation. During the initial 2 weeks after anthesis, hexose levels rise sharply (Ho and Hewitt, 1986; Wang et al., 1993). However, the pattern of hexose accumulation during the later stages of fruit development is not clear, and there appear to be varietal differences. 
Picha (1986) compared glucose and fructose accumulation patterns in small- and large-fruited tomato cultivars and found that only in the former did hexose levels increase during the 2 weeks prior to the breaker stage. Some accounts of tomato fruit development (e.g., Ho et al., 1983; Yelle et al., 1991) do indeed report a significant increase in hexose levels during the final stage of development and some report a constant, though not sharp, rise in hexose levels earlier in fruit maturation but not during the final stage (e.g., Chetelat et al., 1995a; Davies and Kempton, 1975). At present, both cultivar and environment appear to affect hexose accumulation, but further study of these different hexose accumulation patterns is necessary.

During the early stages of fruit development, there is a transient period of starch accumulation, particularly in the columella, the placenta, and the inner and radial portions of the pericarp (Schaffer and Petreikov, 1997a). During maturation, the starch is degraded so that starch levels are nonsignificant in the mature fruit. The fate of the degraded starch has not been studied, but it may function as a reservoir for soluble sugars of the mature fruit (Dinar and Stevens, 1981).

Recent results (Ruan and Patrick, 1995) indicate that a developmental transition occurs, from symplastic to apoplastic unloading into the fruit. The symplastic phase is associated with starch accumulation whereas the apoplastic phase is associated with hexose accumulation. This transition is accompanied by metabolic transitions in sucrose metabolism (Robinson et al., 1988; Schaffer and Petreikov, 1997a; Wang et al., 1993). In the symplastic stage, cytosolic sucrose synthase appears to play a major role in sucrose metabolism, whereas in the apoplastic phase cell-wall invertase seems to function as the key enzyme of sucrose hydrolysis. Accordingly, the recent report that genotypic differences in mature fruit hexose levels may be related to plasmalemma hexose carriers that function during the period of apoplastic unloading (Ruan et al., 1997) is most significant.

Research has focused on the study of carbohydrate metabolism in the tomato fruit, with the goal of applying this knowledge to the selection of genotypes that have a significantly different pattern of fruit carbohydrate accumulation. To this end, progress has been made on three fronts: starch accumulation, sucrose accumulation, and fructose accumulation, and these will be described in the following sections.

\section{STARCH ACCUMULATION}

Dinar and Stevens (1981), in a study comparing six tomato genotypes, first reported the correlation between starch content of the young immature fruit and soluble solids content of the mature fruit. This observation led them to hypothesize that the transiently stored starch could function as a reservoir of carbohydrate that later contributes to the soluble sugar content of the mature fruit. In order to shed light on the metabolism of starch synthesis in tomato fruit, Robinson et al. (1988) studied the activity patterns of sucrose synthase and ADPGlu pyrophosphorylase (ADPGPPase), the initial and one of the final enzymes in sucrose-to-starch metabolism, respectively, in the developing fruit. They reported that both enzymes undergo transient activity patterns, parallel with the transient accumulation of starch. Wang et al. (1993) further established sucrose synthase as the key enzyme responsible for sucrose cleavage during the starch accumulation phase, and correlated its activity with tomato fruit growth and drymatter accumulation.

We recently reported the developmental changes in activities of 10 enzymes in the sucrose-to-starch metabolic pathway (Schaffer and Petreikov, 1997a). Four of the enzymes (sucrose synthase, fructokinase, ADPGPPase, and starch synthase) undergo what appears to be a coordinated developmental loss of activity, temporally parallel to the decrease in starch content. The maximal catalytic activities of these same four enzymes appear to be limiting to the rate of starch synthesis in the developing fruit, whereas the activities of the other enzymes studied are neither temporally correlated with, nor are their observed maximal activities limiting to starch synthesis. The possible limiting activities of two of these enzymes, sucrose synthase and fructokinase, are especially evident when their activities are measured in the presence of physiological levels of fructose, which strongly inhibits both of them (Schaffer and Petreikov, 1997b).
Starch deposition is also under spatial control, as mentioned previously. The outer pericarp tissue, which may comprise $\approx 15 \%$ of the bulk fruit tissue, accumulates little starch (Schaffer and Petreikov, 1997a; Wang et al. 1994); this may be due to the low levels of ADPGPPase activity and ADPGPPase protein in this tissue.

Our conclusion from the study of sucrose-to-starch metabolism in developing tomato fruit is that starch content and, therefore, the carbohydrate reservoir, of the fruit can be increased by increasing

1) the absolute rate of starch synthesis,

2) the relative amount of starch-accumulating tissue, or

3) the period of transient starch accumulation so that starch synthesis continues as the fruit increases in volume.

In order to show the potential contribution of the starch reservoir to soluble sugar levels, a simple calculation will suffice. The carbohydrate reservoir of a medium-sized $(100 \mathrm{~g})$ fruit that reaches a maximum bulk starch concentration of $2 \%$ when the young fruit weighs only 10 $\mathrm{g}$ will be only $200 \mathrm{mg}$, or $0.2 \%$ of its final fresh weight. However, increasing the maximum bulk tissue starch concentration to $3 \%$ and maintaining this level of starch accumulation until the fruit weighs 50 $\mathrm{g}$ would increase this to $1.5 \%$ of final fresh weight.

Indications from the recent development of transgenic tomatoes overexpressing ADPGPPase support the hypothesis that this enzyme is limiting to starch accumulation (Stark et al., 1996). We are presently studying the effect of overexpression of fructokinase in tomato fruit, although increasing fructokinase activity may not concomitantly increase starch levels, since it is only one of the four coordinatedly controlled enzymes, and modulating its activity alone will not necessarily increase the sucrose-to-starch flux. On the other hand, increasing ADPGPPase activity may be successful by itself, since the outer pericarp tissue lacks this enzyme (Schaffer and Petreikov, 1997a) and overexpressing it under the control of a nonspecific promoter may allow the synthesis of starch in $15 \%$ of the bulk tomato tissue, which normally contains low levels of this enzyme.

\section{SUCROSE ACCUMULATION}

Many plant tissues that store large amounts of sugar do so by accumulating sucrose, generally at a later stage of development, as in the cases of melon fruits (Schaffer et al., 1987) and sugar beet roots (Giaquinta, 1979). The advantages of sucrose for high sugar storage are that: 1) it is relatively inactive biochemically, since its metabolism depends on only a few enzymes (Pontis, 1978), and, 2) as a disaccharide, it is a more efficient storage carbohydrate than are monosaccharides, because of osmotic considerations. The fruit of certain wild species of Lycopersicon, those belonging to the green-fruited subgenus Eriopersicon (i.e., L. hirsutum Humb. and Bonpl., L. chmielewskii Rick, Kes., Fob. and Holle, L. peruvianum (L.) Mill.), are characterized by developmental sucrose accumulation (Davies, 1966) and also contain relatively high concentrations of soluble sugars. Therefore, based on the hypothesis that the two characteristics are linked and that sucrose accumulation is causal to high sugar levels, efforts were undertaken to develop sucrose-accumulating tomato genotypes.

The three wild species mentioned above have each been studied with respect to sugar accumulation and activities of sucrose-metabolizing enzyme during fruit development (Miron and Schaffer, 1991; Stommel, 1992; Yelle at al., 1988) and in each of these species, sucrose accumulation was associated with the developmental loss of acid invertase activity, particularly the vacuolar soluble invertase activity (Miron and Schaffer, 1991). The cell-wall-bound invertase activity appears to be maintained even during sucrose accumulation (Miron and Schaffer, 1991), and this led to the hypothesis that sucrose accumulation is part of a sucrose hydrolysis-resynthesis pathway. According to this scheme, apoplastically unloaded sucrose is hydrolyzed by cell-wall invertase, the hexose products are carried across the membrane into the cytosol where the resynthesis of sucrose occurs, and the sucrose is subsequently localized in the vacuole where the absence of acid invertase permits its storage (Dali et al., 1992; Miron and Schaffer, 1991). Our recent results (Miron, Zamski, and Schaffer, unpublished) indicate that, although hydrolysis-resynthesis of sucrose occurs, it is part of the natural turnover of sucrose in the tomato fruit. However, based on short-term uptake studies of asymmetrically 
labelled sucrose, hydrolysis-resynthesis is not the requisite mechanism of sucrose uptake and storage. Rather, sucrose is taken up into the vacuole intact, in spite of the presence of the invertase protein in the cell wall (based on immunolocalization) and the presence in vitro of cell-wall bound invertase activity.

The genetic-biochemical trait of sucrose accumulation has been transferred to L. esculentum, and is controlled by a single recessive gene, termed sucr (Chetelat et al., 1993; Hadas et al., 1995; Stommel and Haynes 1993; Yelle et al., 1991). The traits from L. chmielewskii and $L$. hirsutum are allelic (Chetelat et al., 1993). Molecular markers have been developed to assist the transfer and breeding efforts (Chetelat et al., 1993, 1995a; Hadas et al., 1995) and nearly isogenic sucroseaccumulating tomatoes have been developed by marker-assisted classical breeding techniques (Chetelat et al., 1995a, 1995b). Furthermore, an acid invertase gene has been cloned from tomato, and antisense transgenic tomato plants with decreased acid invertase in the developing fruit have been generated (Klann et al., 1996; Ohyama et al., 1995). These transgenic fruits, in fact, accumulate sucrose.

The comparison of sucrose-accumulating tomatoes with standard hexose-storing tomatoes allows us to determine whether sucrose accumulation is causal to increased sugar content of the fruit. Results from both the classical breeding strategy, as well as the transgenic strategy, clearly indicate that the loss of invertase activity and sucrose accumulation does not per se increase the total sugar content in the fruit. Fruit size is reduced, presumably due to a reduction in water uptake because of osmotic effects, and, therefore, sugar concentration, but not sugar content, is increased (Chetelat et al., 1995b; Klann et al., 1996). The biochemical modification of tomatoes for the trait of sucrose accumulation does not appear to causally modify the sugar import or carbon economy of the fruit. Rather, it modifies the partitioning of sugars in the fruit, with concomitant effects on fruit-water relations.

\section{FRUCTOSE ACCUMULATION}

Since fructose is twice as sweet as glucose (Biester, 1925), it was reasoned that increasing the fructose : glucose ratio of the hexose content in mature fruit could affect its taste. As mentioned before, $L$. esculentum genotypes are characterized by nearly equimolar ratios of fructose and glucose. A high frucose : glucose ratio had been previously reported in the sucrose-accumulating wild species (Davies, 1966), but was largely ignored because hexose sugars comprise only a small proportion of the total soluble sugars in these species. Stommel and Haynes (1993) studied the inheritance of the trait of high fructose : glucose ratio in the $\mathrm{F}_{2}$ progeny of the interspecific L. esculentum $\times L$. hirsutum cross and suggested that the trait is controlled by at least two genes. However, population skewing in early interspecific progeny can modify expected ratios of segregation (Chetelat et al., 1993; Zamir and Tadmor, 1986).

We have developed stable, true-breeding, low-sucrose, high fructose : glucose ratio (F:G) tomato lines in advanced backcross populations derived from selected $\mathrm{F}_{4}$ plants from an initial interspecific $L$. esculentum $\times$ L. hirsutum cross. When these lines were used as the high $\mathrm{F}: \mathrm{G}$ parent, the trait of high $\mathrm{F}: \mathrm{G}$ was controlled by a single, major, incompletely dominant gene, termed Fgr (Levin et al., 1993). The development of a molecular marker for the trait allowed us to classify individual plants in the segregating $\mathrm{F}_{2}$ population as $\mathrm{HH}, \mathrm{HE}$, or $\mathrm{EE}$, for the hirsutum and esculentum alleles, respectively. Although the hirsutum allele confers a higher F:G ratio, it does not seem to influence the total hexose content, but rather affects only the partitioning of hexoses within the fruit (unpublished). This is reminiscent of the results from the transfer of the sucr gene described previously, in which total sugar is not increased but the sugar profile is modified.

Further study of this material should contribute to our understanding of hexose metabolism in tomato fruit. Since the trait of high F:G is observed in mature fruit and not in young, starch-synthesizing fruit, invertase hydrolysis rather than sucrose synthase cleavage is probably the initial step in the metabolism of translocated sucrose (Robinson et al., 1988; Schaffer and Petreikov, 1997a). Metabolic pathways leading to fructose and its potential accumulation may involve hexose phosphorylation and the isomerization of hexose phosphates. According to this model, the balance of hexose kinase and hexose-phosphate phosphatase activities, in conjunction with the isomerase reaction, may affect the ratio of fructose : glucose.

The fructokinase reaction in tomato fruit has recently begun to attract research attention (Kanayama et al., 1997; Martinez-Baranas and Randall, 1996; Schaffer and Petreikov, 1997b), partially because of its involvement in transient starch accumulation. Two genes for tomato fructokinase have recently been cloned (Kanayama et al., 1997). One of the enzymes (Frk2) appears to be associated primarily with the starch-synthesis phase, based on developmental expression patterns. The second enzyme (Frk1) is expressed throughout fruit development and perhaps plays a role in hexose partitioning in the mature fruit. With regard to the phosphatase reactions, very little is known. While nonspecific phosphatases can act on hexose-P (Leigh and Walker, 1980; Park and van Etten, 1986), specific hexose-P phosphatases have not been studied in plants. The possible contribution of the phosphoglucoisomerase reaction to the control of F: $\mathrm{G}$ is not clear since, as a reversible reaction operating near its equilibrium, its substrate and product concentrations are probably not determined by this metabolic step.

In summary, research and breeding efforts are being carried out with the goal of improving tomato fruit taste. The strategy adopted is to develop tomato genotypes with modified fruit carbohydrate metabolism, which may impact on the sugar composition of the mature fruit. A clearer understanding of the carbon economy and carbohydrate metabolism in the tomato fruit should improve the chances of success in this endeavor.

\section{Literature Cited}

Biester, A.M. 1925. Carbohydrate studies. I. Relative sweetness of pure sugars. Amer. J. Physiol. 73:387-400.

Chetelat, R.T., J.W. DeVerna and A.B. Bennett. 1995a. Introgression into tomato (Lycopersicon esculentum) of the Lycopersicon chmielewskii sucrose accumulator gene (sucr) controlling fruit sugar composition. Theor. Appl. Genet. 91:327-333.

Chetelat, R.T., J.W. DeVerna, and A.B. Bennett. 1995b. Effects of the Lycopersicon chmielewskii sucrose accumulator gene (sucr) on fruit yield and quality parameters following introgression into tomato. Theor. Appl. Genet. 91:334-339.

Chetelat, R.T., E. Klann, J.W. DeVerna, S. Yelle, and A.B. Bennett. 1993. Inheritance and genetic mapping of fruit sucrose accumulation in Lycopersicon chmielewskii. Plant J. 4:643-650.

Dali, N., D. Michaud, and S. Yelle. 1992. Evidence for the involvement of sucrose phosphate synthase in the pathway of sugar accumulation in sucrose-accumulating tomato fruit. Plant Physiol. 99:434-438.

Davies, J.N. 1966. On the occurrence of sucrose in the fruit of some species of Lycopersicon. Nature 209:640-641.

Davies, J.N. and G.E. Hobson. 1981. The constituents of tomato fruit-The influence of environment, nutrition and genotype. CRC Crit. Rev. Food. Sci. Nutr. 15:205-280.

Davies, J.N. and R.J. Kempton. 1975. Changes in the individual sugars of tomato fruit during ripening. J. Sci. Food Agr. 26:1103-1110.

Dinar, M. and M.A. Stevens. 1981. The relationship between starch accumulation and soluble solids content of tomato fruit. J. Amer. Soc. Hort. Sci. 106:415-418.

Giaquinta, R.T. 1979. Sucrose translocation and storage in the sugar beet. Plant Physiol. 63:828-832.

Hadas, R., A.A. Schaffer, D. Miron, M. Fogelman, and D. Granot. 1995. PCRgenerated molecular markers for the invertase gene and sucrose accumulation in tomato. Theor. Appl. Genet. 90:1142-1148.

Ho, L.C. 1996a. Tomato, p. 709-728. In: E. Zamski and A.A. Schaffer (eds.). Photoassimilate distribution in plants and crops: Source-sink relationships. Marcel Dekker, New York.

Ho, L.C. 1996b. The mechanism of assimilate partitioning and carbohydrate compartmentation in fruit in relation to the quality and yield of tomato. $\mathrm{J}$. Expt. Bot. 47:1239-1243.

Ho, L.C. and J.D. Hewitt. 1986. Fruit development, p. 201-240. In: J.G. Atherton and J. Rudich (eds.). The tomato crop. Chapman and Hall, London.

Ho, L.C., V. Sjut, and G.V. Hoad. 1983. The effect of assimilate supply in fruit growth and hormone levels in tomato plants. Plant Growth Regulat. 1:155171.

Kanayama, Y., N. Dai, D. Granot, M. Petreikov, A. Schaffer, and A.B. Bennett. 1997. Divergent fructokinase genes are differentially expressed in tomato. Plant Physiol. 113:1379-1384.

Klann, E., B. Hall, and A.B. Bennett. 1996. Antisense acid invertase (TIV1) 
gene alters soluble sugar composition and size in transgenic tomato fruit. Plant Physiol. 112:1321-1330.

Leigh, R.A. and R.R. Walker. 1980. ATPase and acid phosphatase activities associated with vacuoles isolated from storage roots of red beet (Beta vulgaris L.). Planta 150:222-229.

Levin, I., N. Gilboa, E. Yeselson, S. Shen, and A.A. Schaffer. 1999. Fgr, a major locus that modulates the fructose to glucose ratio in mature tomato fruits. Theor. Appl. Genet. (In press.)

Martinez-Barajas, E. and D.D. Randall. 1996. Purification and characterization of fructokinase from developing tomato (Lycopersicon esculentum Mill.) fruits. Planta 199:451-458.

Miron, D. and A.A. Schaffer. 1991. Sucrose phosphate synthase, sucrose synthase and acid invertase activities in developing fruit of Lycopersicon esculentum Mill. and the sucrose accumulating Lycopersicon hirsutum Humb. and Bonpl. Plant Physiol. 95:623-627.

Ohyama, A., H. Ito, T. Sato, S. Nishimura, T. Imai, and M. Hirai. 1995. Suppression of acid invertase activity by antisense RNA modifies the sugar composition of the fruit. Plant Cell Physiol. 36:369-376.

Park, H.-S.C. and R.L. vanEtten. 1986. Purification and characterization of homogeneous sunflower seed acid phosphatase. Phytochemistry 25:351357

Picha, D.H. 1986. Effect of harvest maturity on the final composition of cherry and large fruited tomato cultivars. J. Amer. Soc. Hort. Sci. 111:723-727.

Pontis, H.G. 1978. On the scent of the riddle of sucrose. Trends Biochem. 3:137-139.

Robinson, N.L., J.D. Hewitt, and A.B. Bennett. 1988. Sink metabolism in tomato fruit. I. developmental changes in carbohydrate metabolizing enzymes. Plant Physiol. 87:727-730.

Ruan, Y.-L. and J.W. Patrick. 1995. The cellular pathway of postphloem sugar transport in developing tomato fruit. Planta 196:434-444.

Ruan, Y.-L., J.W. Patrick, and C. Brady. 1997. Protoplast hexose carrier activity is a determinate of genotypic difference in hexose storage in tomato fruit. Plant Cell Environ. 20:341-349.

Schaffer, A.A., B. Aloni, and E. Fogelman. 1987. Sucrose metabolism and accumulation in developing fruit of Cucumis. Phytochemistry 26:18831887.
Schaffer, A.A. and M. Petreikov. 1997a. Sucrose-to-starch metabolism in tomato fruit undergoing transient starch accumulation. Plant Physiol. 113:73-746.

Schaffer, A.A. and M. Petreikov. 1997b. Inhibition of fructokinase and sucrose synthase in young tomato fruit undergoing transient starch synthesis by cytosolic levels of fructose. Physiol. Plant. 101:800-806.

Stark, D.M., G.F. Barry, and G.M. Kishore. 1996. Improvement of fruit quality traits through enhancement of starch synthesis. Ann. New York Acad. Sci. 792:26-36.

Stevens, M.A., A.A. Kader, and M. Albright-Holton. 1977a. Intercultivar variation in composition of locular and pericarp portions of fresh market tomatoes. J. Amer. Soc. Hort. Sci. 102:689-697.

Stevens, M.A., A.A. Kader, M. Albright-Holton, and M. Algazi. 1977b. Genetic variation for flavor and composition in fresh market tomatoes. J. Amer. Soc. Hort. Sci. 102:680-689.

Stommell, J.R. 1992. Enzymatic components of sucrose accumulation in the wild tomato species Lycopersicon peruvianum. Plant Physiol. 99:324-328.

Stommell, J.R. and K.G. Haynes. 1993. Genetic control of fruit sugar accumulation in a Lycopersicon esculentum $\times$ L. hirsutum cross. J. Amer. Soc. Hort. Sci. 118:859-863.

Walker, A.J. and L.C. Ho. 1977. Carbon translocation in the tomato: Carbon import and fruit growth. Ann. Bot. 41:813-823.

Wang, F., A. Sanz, M.L. Brenner, and A.G. Smith. 1993. Sucrose synthase, starch accumulation and tomato fruit sink strength. Plant Physiol. 101:321327.

Wang, F., A.G. Smith, and M.L. Brenner. 1994. Temporal and spatial expression of sucrose synthase during tomato fruit development. Plant Physiol. 104:535-540.

Yelle, S., R.T. Chetelat, M. Dorais, J.W. Deverna, and A.B. Bennett. 1991. Sink metabolism in tomato fruit. IV. Genetic and biochemical analysis of sucrose accumulation. Plant Physiol. 95:1026-1035.

Yelle, S., J.D. Hewitt, N.L. Robinson, S. Damon, and A.B. Bennett. 1988. Sink metabolism in tomato fruit. III. Analysis of carbohydrate metabolism in a wild species. Plant Physiol. 87:737-740.

Zamir, D. and Y. Tadmor. 1986. Unequal segregation of nuclear genes in plants. Bot. Gaz. 147:355-358. 